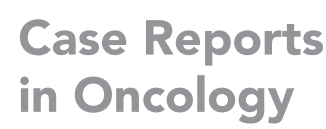

Case Reports

in Oncology

\title{
A Case of Squamous Cell Carcinoma of the Breast with Low-Grade Adenosquamous Carcinoma
}

\author{
Nozomi Kashu $^{a}$ Shoji Ourab ${ }^{b}$ Hideyuki Yoshidac $^{c}$ Eisei Nishinod \\ Shinichiro Makimoto ${ }^{a}$ \\ a Department of Surgery, Kishiwada Tokushukai Hospital, Kishiwada, Japan; ${ }^{b}$ Division of \\ Breast Surgery, Kishiwada Tokushukai Hospital, Kishiwada, Japan; ' $Y o s h i d a$ Breast Home \\ Clinic, Ohtsu, Japan; dDivision of Pathology, Kishiwada Tokushukai Hospital, Kishiwada, \\ Japan
}

\section{Keywords}

Breast cancer · Low-grade adenosquamous cell carcinoma - Squamous cell carcinoma

\begin{abstract}
A 68-year-old woman with a breast mass was referred to our hospital. Imaging studies showed an oval well-defined mass, $1.3 \mathrm{~cm}$ in size, in her left lower outer quadrant of the breast. Core needle biopsy with immunohistochemical staining showed atypical spindle cells forming solid nests with necrosis and papillary lesions, leading to the tentative and pre-operative diagnosis of invasive ductal carcinoma. Due to the absence of daughter nodules, extensive ductal spread, and lymphadenopathy on imaging evaluation, the patient underwent breast-conserving surgery and sentinel node biopsy, resulting in negative surgical margins and no lymph node involvement. Post-operative pathological examination showed triple negative atypical cells with squamous differentiation, squamous cell carcinoma (SCC), with cystic parts and a small amount of low-grade adenosquamous cell carcinoma (LGASC), both encompassing the cystic parts in a contiguous fashion. No cases with synchronous SCC and LGASC in the breast have been reported to date. An etiologic correlation between SCC and LGASC should be further evaluated.

\section{Introduction}

Breast cancer arises from mammary gland epithelium, naturally exhibiting adenocarcinomatous features. Rare cases, however, partially or totally present a non-glandular malignant growth pattern, called metaplastic breast carcinoma (MpBC) [1-5]. MpBC generally has 


\section{Case Reports in Oncology}

Fig. 1. Mammography of the left breast showed a mass (arrow) with partially ill-defined borders in the lower (a; MLO view) and outer (b; CC view) quadrant of the breast.

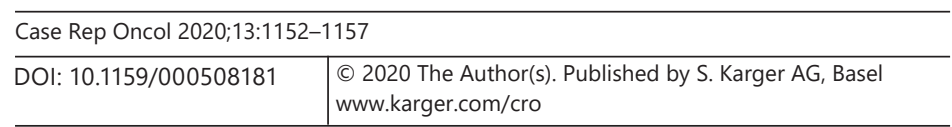

Kashu et al.: SCC of the Breast with Low-Grade Adenosquamous Carcinoma
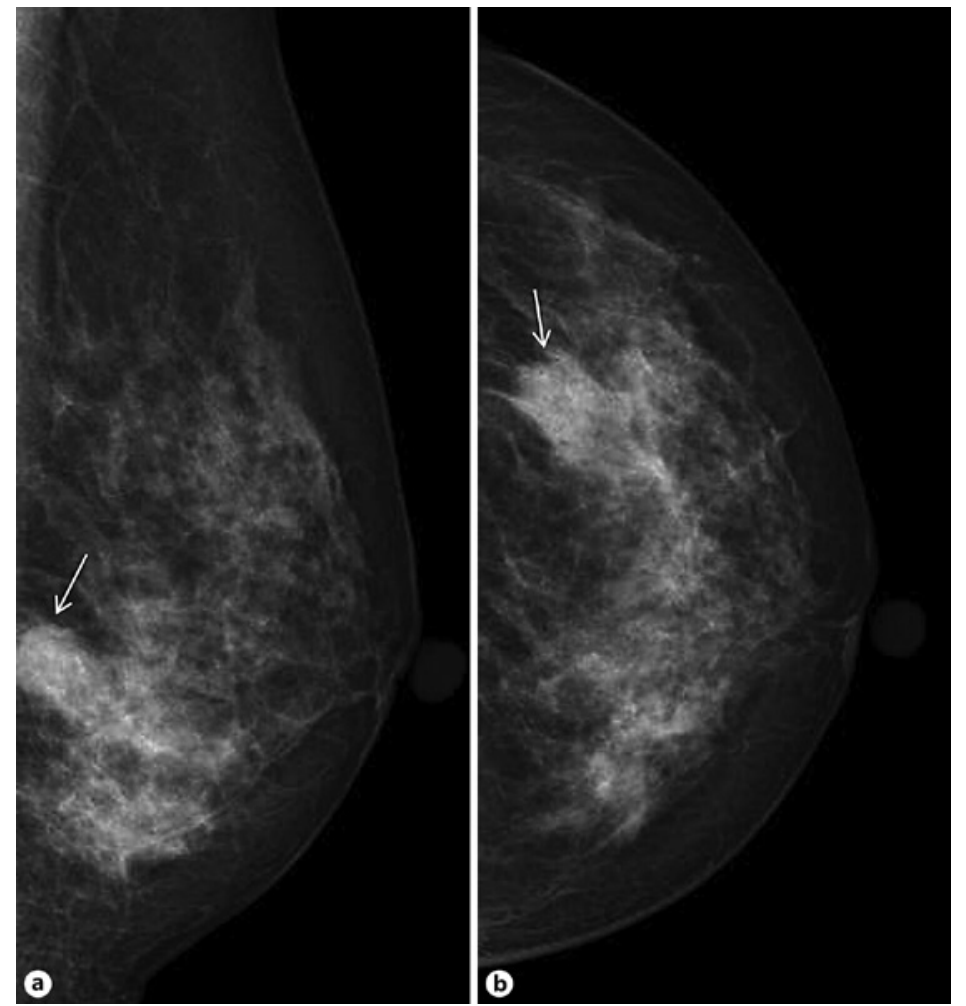

squamous and/or mesenchymal components $[1,2]$. The word mesenchymal includes sarcomatous components such as spindle, cartilaginous, osseous, and rhabdomyomatous malignant cells.

Squamous cell carcinoma (SCC) of the breast [6, 7], accounting for about $0.1-0.3 \%$ of all breast cancer cases, is a malignancy with more than $90 \%$ of malignant components showing squamous differentiation. MpBC including SCC generally has triple negative features. In addition, SCC poses a unique clinical manifestation presumably due to the desquamative nature of the multilayered malignant squamous cells. SCC of the breast, therefore, has highly degenerative cystic parts as does the cavity often found in lung SCC.

Low-grade adenosquamous carcinoma (LGASC) of the breast [8-10] is another rare and distinct subtype of $\mathrm{MpBC}$, being morphologically very similar to LGASC of the skin. MpBC generally shows poorer prognosis than invasive ductal carcinoma and invasive lobular carcinoma [4]. However, it is well known that the prognosis of LGASC is better than those of invasive ductal and lobular carcinomas.

We herein report an extremely rare case of SCC of the breast with LGASC.

\section{Case Report}

A 68-year-old woman with a breast mass was referred to our hospital. Mammography showed a mass with amorphous microcalcifications in her left lower outer quadrant of the breast (Fig. 1). Ultrasonography showed an oval tumor measuring $1.3 \mathrm{~cm}$ in size with welldefined margins, enhanced posterior echoes, and heterogenous low-level internal echoes. Doppler mode ultrasound showed the blood flow into and around the tumor (Fig. 2). Core needle biopsy showed atypical spindle cells forming solid nests with necrosis and papillary 


\section{Case Reports in Oncology}

Case Rep Oncol 2020;13:1152-1157

DOI: $10.1159 / 000508181$

(c) 2020 The Author(s). Published by S. Karger AG, Basel www.karger.com/cro

Kashu et al.: SCC of the Breast with Low-Grade Adenosquamous Carcinoma
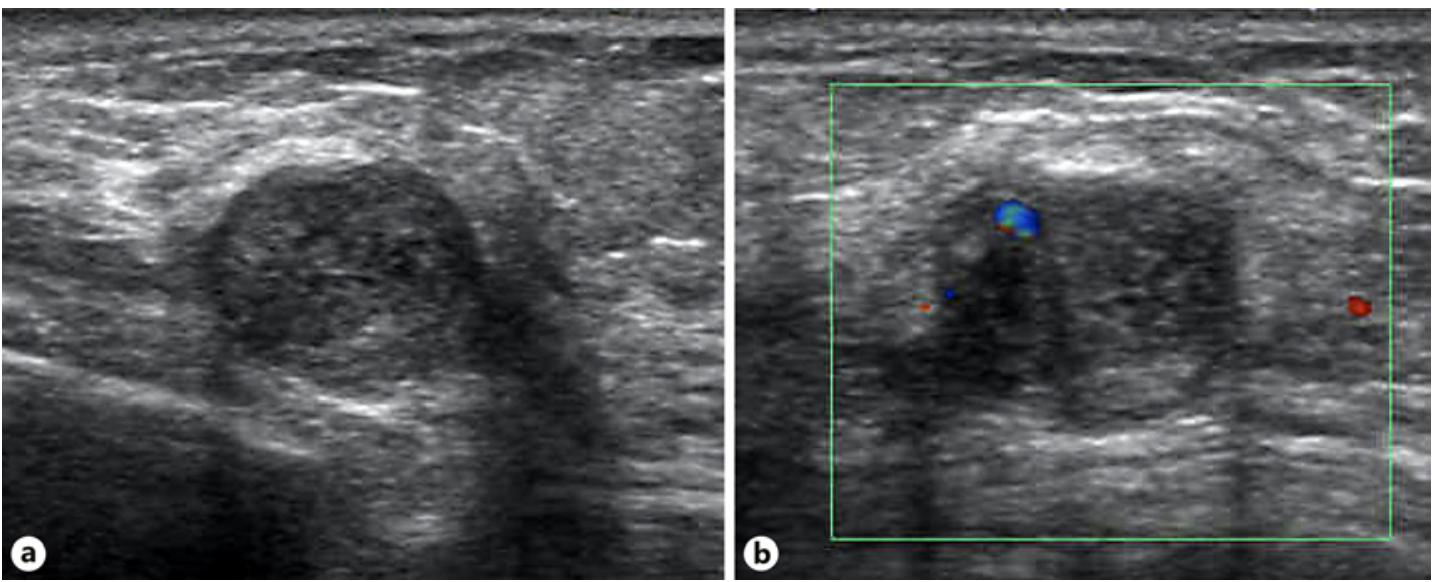

Fig. 2. Ultrasonography showed a well-demarcated mass with heterogenous and low-level internal echoes (a) and blood flow into the tumor (b).
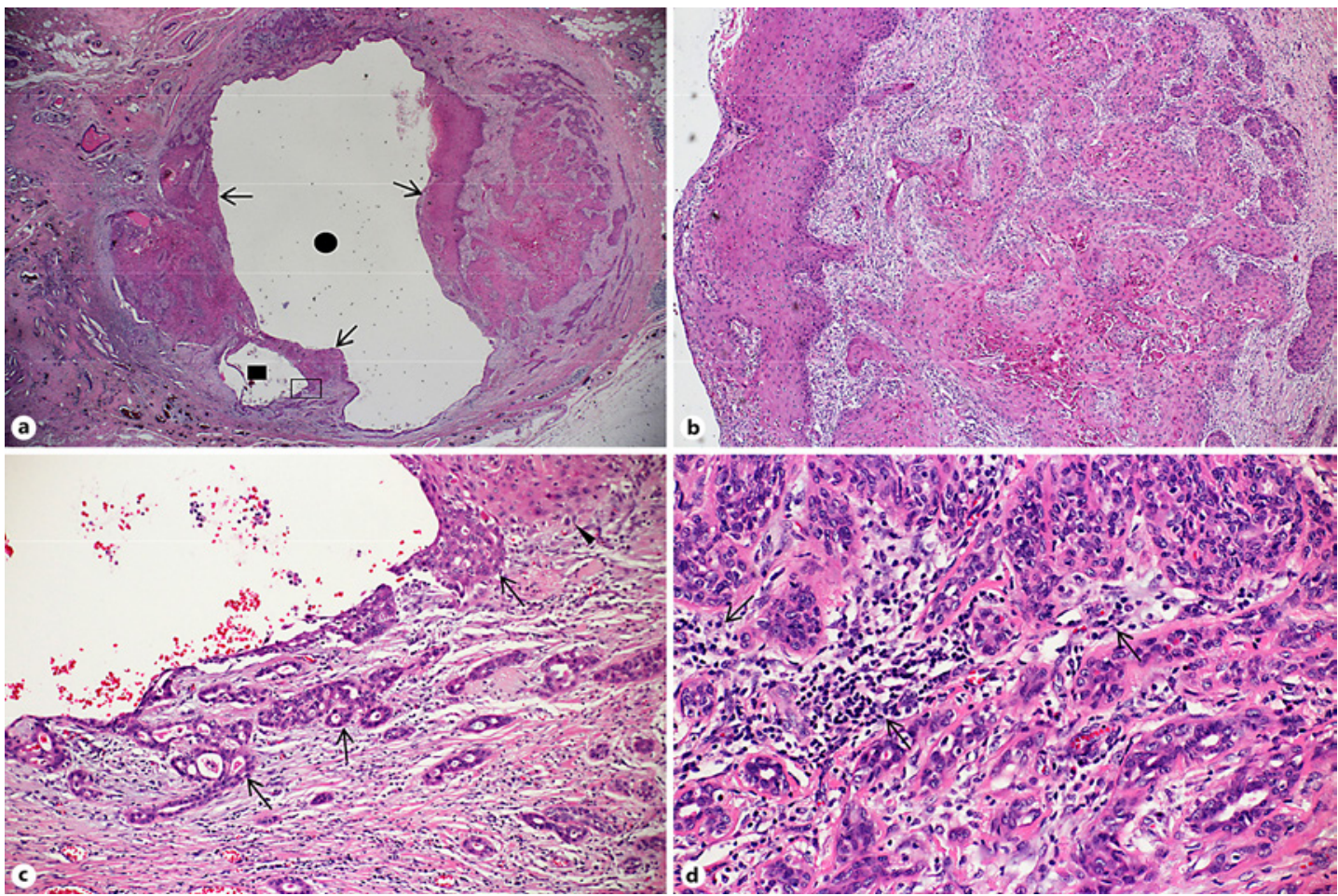

Fig. 3. Pathological findings. Low magnification (a) showed an oval tumor with centrally located large (closed circle) and peripherally located small (closed square) cystic parts encompassed by atypical cells. In addition to SCC cell layers surrounding the cystic parts, SCC were also observed in a cobblestone appearance (b). Magnified view (c) of the open square in a showed atypical cells with bland nuclei, small glandular structure, and squamous differentiation (LGASC; arrows) contiguously to SCC (arrowhead). Diffuse prominent lymphocytic infiltrates (arrows) were observed around the LGASC cells (d).

lesions, leading to the tentative diagnosis of breast cancer. Immunohistochemical staining showed the papillary lesions to be CD10 and p63 negative and the solid nests to be CAM5.2 and $A E 1 / 3$ positive, suggesting that the tumor was an invasive carcinoma. Under the diagnosis 
of presumed invasive ductal carcinoma, due to the absence of daughter nodules, extensive ductal spread, and lymphadenopathy on imaging examinations, the patient underwent partial mastectomy and sentinel node biopsy, resulting in negative surgical margins and no lymph node metastases. Pathological examination showed an invasive tumor containing cystic parts, locating mainly in the center of the tumor, encompassed by atypical cells with squamous epithelial layers, partially protruding into the degenerative cyst. The tumor cells also proliferated coalescently to the peripheral stroma and showed cobblestone appearance with keratinization and surrounding desmoplastic stroma (Fig. 3a, b), leading to the diagnosis of moderately differentiated SCC. Just outside and contiguously to the SCC, LGASC with bland nuclei, tubule formation, and diffuse lymphocytic infiltrates were observed (Fig. 3c, d). Immunostaining was negative for all estrogen receptor, progesterone receptor, and human epidermal growth factor receptor type 2 and showed 14\% positivity for the Ki-67 labeling index. The pathological tumor size was $8 \mathrm{~mm}$ (pT1b), and no vessel and lymphatic permeation was observed. The patient was discharged from hospital after a week without any events. We recommended adjuvant therapy, that is, radiotherapy \pm chemotherapy, to the patient. The patient, however, refused to receive adjuvant therapy and has only been followed up.

\section{Discussion}

Mammary glandular tissue is susceptible to squamous metaplasia, often found in the glandular epithelium, especially in the myoepithelium [6], of cysts, gynecomastia, papilloma, and even in the reparative epithelium at a biopsy site [1]. An in vitro study using human mammary gland explants showed that squamous metaplasia was observed in untreated cultured explants from postmenopausal women but not in those from premenopausal women [11]. An epidemiological study using the National Cancer Institute's Surveillance, Epidemiology and End Results Registry showed that the median age of patients with SCC of the breast was 67 years [7], very close to that of our case.

SCC of the breast is generally detected as a palpable tumor with circumscribed borders, cystic parts, and triple negative features, well corresponding to those of the tumor in this case. The etiology of cystic parts is generally ascribed to the rapid growth nature of the SCC, leading to larger tumor sizes than those of triple-negative invasive ductal carcinomas of no special type. In this case, the tumor size was small with $1.3 \mathrm{~cm}$, and the Ki-67 labeling index was low with $14 \%$. Like daily skin turnover, the stratum corneum naturally exfoliates when its thickness exceeds a certain level. In this case, degenerative cysts were surrounded by malignant hyperkeratotic cell layers with very little intermingling of stromal cells and glandular components, presumably resulting in the degenerative cystic formation despite the relatively small tumor size and low Ki-67 labeling index.

LGASC develops much less frequently in the breast than SCC and has features such as infiltrative glands with bland nuclei, focal squamoid differentiation, desmoplastic stroma, and lymphoid aggregates. We could not identify any apparent lymphoid aggregates in this case, making us hesitant to make a definitive diagnosis of LGASC. However, we made a final diagnosis of LGASC both due to the bland and uniform nuclei of the lesions and the presence of widespread lymphoid infiltrates around the LGASC cells.

To our best knowledge, no reports with coexistence of SCC and LGASC, not only in a contiguous fashion like in this case but also even in the same breast, have been published. In this case, a small amount of LGASC encompassed the SCC in a contiguous fashion, possibly suggesting some correlation between SCC and LGASC. LGASC of the breast has been highlighted as an $\mathrm{MpBC}$ with favorable prognosis and significant correlation with radial scar/ complex sclerosing lesion [12], often showing a stellate tumor shape markedly different from

\section{Karger ${ }^{\prime}=$}


that of SCC with relatively clear borders. A small amount of LGASC, therefore, might have been ignored or overlooked thus far, even if coexisting with SCC. In fact, we did notice the SCC but not the LGASC before and during operation.

In conclusion, we cannot speculate why SCC and LGASC coexisted contiguously in our case. Correlation between LGASC and SCC should further be examined immunohistochemically and genetically [10].

\section{Acknowledgements}

We thank Dr. Masafumi Kurozumi (Department of Breast Pathology, Kameda Medical Center) for his informative advice on the pathological evaluation.

\section{Statement of Ethics}

We have reported this case in compliance with the Declaration of Helsinki. Informed consent was obtained from the patient for the publication of the clinical data.

\section{Conflict of Interest Statement}

The authors have no conflicts of interest to declare.

\section{Funding Sources}

Not applicable.

\section{Author Contributions}

N.K. contributed the design of the report and collected the data. S.O. drafted the manuscript. H.Y. diagnosed and operated the patient. E.N. performed the pathological evaluation. S.M. revised the manuscript. All authors have read and approved the final version of the manuscript.

\section{References}

1 Rosen PP. Rosen's breast pathology. Philadelphia: Lippincott Williams\&Wilkins;. p. 424-44.

2 Reis-Fiho JS, Gobbi H, McCart RAE, Rakha EA, Shin SJ, Sotiriou C, et al. Metaplastic carcinoma. Breast tumours. WHO classification of tumours. 5th ed. Lyon: IARC; 2019. p. 134-8.

3 Krings G, Chen YY. Genomic profiling of metaplastic breast carcinomas reveals genetic heterogeneity and relationship to ductal carcinoma. Mod Pathol. 2018;31(11):1661-74

4 El Zein D, Hughes M, Kumar S, Peng X, MS, Jabbour H, et al. Metaplastic carcinoma of the breast is more aggressive than triple-negative breast cancer: a study from a single institution and review of literature. Clin Breast Cancer. 2017;17(5):382-91.

5 McKinnon E, Xiao P. Metaplastic carcinoma of the breast. Arch Pathol Lab Med. 2015;139(6):819-22.

6 Kokufu I, Yamamoto M, Fukuda K, Kitano H, Horioka Y, Tsuji M. Squamous cell carcinoma of the breast: three case reports. Breast Cancer. 1999;6(1):63-8.

7 Yadav S, Yadav D, Zakalik D. Squamous cell carcinoma of the breast in the United States: incidence, demographics, tumor characteristics, and survival. Breast Cancer Res Treat. 2017;164(1):201-8. 
8 Wu HB, Zhang, AL, Wang W, Li H. Expression of hormone receptors in low-grade adenosquamous carcinoma of the breast: a case report. Medicine (Baltimore) 2017;96(46):e8785.

9 Handa P, Khader SN, Buchbinder SS, Guelfguat M. Low-Grade adenosquamous carcinoma of the breast developing around a localization wire fragment. Lab Med. 2015;46(3):241-7.

10 Geyer FC, Lambros MB, Natrajan R, Mehta R, Mackay A, Savage K, et al. Genomic and immunohistochemical analysis of adenosquamous carcinoma of the breast. Mod Pathol. 2010;23(7):951-60.

11 Heffelfinger SC, Miller MA, Gear R, Devoe G. Staurosporine-induced versus spontaneous squamous metaplasia in pre- and postmenopausal breast tissue. J Cell Physiol. 1998;176(2):245-54.

12 Wilsher MJ, Owens TW, Allcock RJ. Next generation sequencing of the nidus of early (adenosquamous proliferation rich) radial sclerosing lesions of the breast reveals evidence for a neoplastic precursor lesion. J Pathol Clin Res. 2017;3(2):115-22. 